\title{
Assessment of surface roughness for the analysis of the water vapour condensation process
}

\author{
A. J. Klemm ${ }^{1}$, P. Klemm ${ }^{2}$ \& I. Ibrahim ${ }^{2}$ \\ ${ }^{1}$ Glasgow Caledonian University, UK \\ ${ }^{2}$ Technical University of Lodz, Poland
}

\begin{abstract}
This paper presents part of a larger study on water vapour condensation processes on solid surfaces. The strong relationship between the rapid growth of water vapour condensate and the roughness of the surface was the inspiration to undertake this study. A clear need was perceived to address the problem of assessment of the surface properties, selection of parameters for the analysis and the most appropriate experimental methods. An attempt was made here to review the existing methods of analysis of the geometrical microstructure of surfaces. The paper presents experimental results obtained for glass surfaces.

Keywords: cementitious materials, measurements of surface roughness, condensation process.
\end{abstract}

\section{Introduction}

Moisture performance within buildings and their components is a research area of growing international importance due to the devastating effects which moisture can inflict on building envelopes. Interstitial condensation, high moisture contents and the consequent accumulation of water within a structure can cause a dramatic deterioration in the performance and integrity of building materials [1]. The external surfaces of engineering structures often have lower temperatures than the surrounding environment and in the case of occurrence of high relative humidity of air they are often subjected to water vapour condensation and consequently wetting. Both processes - water vapour condensation and water sorption - are very complex and depend on a number of 
factors, primarily surface properties and environmental conditions [2]. Due to its nature it requires therefore non-standard testing and methods of analysis.

The investigations previously undertaken by the authors give clear evidence of a strong relationship between the rapid growth of water vapour condensate and the roughness of the surface and lead to the conclusion that the condensation process can be modified by design of the geometrical microstructure of surfaces $[3,4]$. A clear need is perceived to address the problem of assessment of the surface properties, selection of parameters for the analysis and the most appropriate experimental methods.

\section{Water vapour condensation on solid surfaces}

Assuming lack of chemical interactions between water molecules and solid surfaces, it is possible to simplify the problem to physical absorption. During the condensation process two clearly distinguished phases can be observed spontaneous nucleus creation and growth of a new phase. Nucleus creation is associated with the change of Gibbs energy $\Delta \mathrm{G}$, which in turn consists of two elements: volumetric energy $\Delta \mathrm{G}_{\mathrm{v}}$ and superficial energy $\Delta \mathrm{G}_{\mathrm{s}}$. The second parameter is critical radius of a nucleus. During the phase transition the unstable phase with higher thermodynamic potential disappears and the new phase with lower potential is created. It is evident that the nuclei are stable when $\Delta \mathrm{G}$ is decreasing e.g. after reaching their critical size $R_{c r}$. If the thermodynamic potential of vapour is lower, then liquid is not usually formed. The initiation of a spontaneous phase transition may occur when the nuclei of new phase reach their critical size.

The difference between the potential $\Delta \mathrm{G}_{\mathrm{v}}$ of the original phase and volumetric potential of new created phase does not change in a case of homogeneous nucleation, where:

$$
\begin{gathered}
R_{c r}=-\frac{2 \gamma}{\Delta G_{v}} \\
\Delta \mathrm{G}_{\mathrm{cr}}=\frac{16 \pi \gamma^{3}}{3\left(\Delta \mathrm{G}_{\mathrm{v}}\right)^{2}}
\end{gathered}
$$

where $\gamma$ is surface tension.

The increment of the thermodynamic potential that is necessary for the creation of critical nucleus on the plane surface for the contact angle $\Theta$ is given by equation:

$$
\Delta \mathrm{G}_{\mathrm{cr}}=\frac{16 \pi \gamma^{3}}{3\left(\Delta \mathrm{G}_{\mathrm{v}}\right)^{2}} \cdot 1 / 4 \cdot(2+\cos \Theta)(1-\cos \Theta)^{2}
$$

In a case of spherical surface maximum value of free energy are given by the following equation:

$$
\Delta \mathrm{G}_{\mathrm{cr}}=\frac{16 \pi \gamma^{3}}{3\left(\Delta \mathrm{G}_{\mathrm{v}}\right)^{2}} \mathrm{~S}(\Theta, \Psi)
$$


where $\mathrm{S}$ is a coefficient dependent on wetting angle $\Theta$ and surface refraction angle $\Psi$.

\section{Experimental methods}

\subsection{Contact method}

Contact method is based on determination of numerical values of parameters of roughness profile as well as its presentation in a form of profilogram of known vertical and horizontal magnification. In the experiment a needle of a known geometry, moving along the surface with a constant speed is used. Its vertical displacements are converted into electrical signals. These amplified signals are then recorded in a form of profilogram. Measuring needle is made in form of a cone ended with a spherical cap of a radius $r$. Application of measuring needle of certain value of radius determine the level of accuracy of the profile representation. In the presented investigation a needle of radius $r_{i}=5 \mu \mathrm{m}$ has been used, though precise measurement of very smooth surfaces require smaller radius of $r_{i}=1 \mu \mathrm{m}$. The vertical angle of a needle was $90^{\circ} \pm 5^{\circ}$. Shape of the needle affects the applied pressure during the measurement. Contact deformations between needle and peaks of the rough surface, due to small forces applied, have an elastic character and have values of order of hundredth or tenth of $\mu \mathrm{m}$. For the purpose of this investigation profile-meter Rank Taylor Hobson Leicester T85001 has been used to determine the following parameters: $R_{a}, R_{q}$, $R_{t m}, R_{p m}$. Accuracy of the equipment was in a rage of $\pm 2 \%$, its resolution $0.01 \mu \mathrm{m}$, range of vertical displacements $-8 \mathrm{~mm}$ and the range of lateral displacements $-120 \mathrm{~mm}$.

\subsection{Optical method (laser method)}

As widely published before, light emitted by laser can be successfully used in the studies and metrology of surface roughness [5-7]. The theory of the diffraction of electromagnetic radiation by the rough surface can be used to establish the relationships between the surface parameters and the field parameters of the reflected electromagnetic wave (intensity of reflected and dissipated light, indicatrix). The most general model of surface from the practical point of view is the one with roughness parameters treated as random process. The solution of such a problem simplifies to determination of random characteristics of the dissipated field of electromagnetic wave.

The theory of dissipation of electromagnetic field by the rough surface was comprehensively presented by Beckmann and Spizzichino [8]. The most commonly adopted roughness parameters are: the mean arithmetic deviation of profile $R_{a}$, the mean square deviation of profile $\sigma$ and the length of correlation $T$.

The choice of the method for the determination of parameters of the reflected (dissipated) electromagnetic wave depends on the boundary conditions associated with geometry of the surface micro-roughness and the length of electromagnetic wave. For the need of this study the principles of the Kirchoff 
method have been adopted. The intensity of reflected light in a mirror direction can be therefore determined from the equation:

$$
I_{Z}=r\left(\theta_{1}\right) \cdot I_{o} \exp \left(-\frac{16 \pi^{2} \sigma^{2}}{\lambda^{2}} \cos ^{2} \Theta_{1}\right)
$$

where: $r\left(\Theta_{1}\right)$ is the coefficient of reflection from ideally smooth surface

$I_{0}$ is the intensity of light incidencing at the angle $\Theta_{1}$.

If the component of the diffusive reflection (in mirror direction) is small compared to mirror component then the following equation can be obtained from:

$$
\sigma=\frac{\lambda}{4 \pi \cos \Theta}
$$

Therefore the estimation of such an angle $\Theta$ for which the mirror component is not seen on the background of diffusive component, enable determination of the mean deviation of profile $\sigma$.

\subsection{SEM method (modulation Y)}

It is well known fact that media heated up to a temperature above absolute zero emit radiation. The laws describing these phenomena do apply only to black body, which is capable of maximum emittance regardless direction and length of wave. This ability is called emissivity and for black body is equal one in any direction and any wavelength. For the real bodies emissivity is always smaller than one and may be different in different directions and wavelengths. Mostly measured and published is total emissivity in the normal direction $\mathrm{E}_{\mathrm{T}, \mathrm{n}}$. Technical black body (model of black body) is usually built of metals, heat resisting alloys and ceramics. Any body in natural environment has total emissivity to semi space $\mathrm{E}_{\mathrm{T}}$ smaller than one. In order to obtain effective emisssivity $\mathrm{E}_{\mathrm{T}, \mathrm{E}}$ from the emitting surface of the technical black body very close to unity, the effect of cavity has been applied. The open area of cavity (emitting opening) $A_{o}$ has much grater effective emissivity $\mathrm{E}_{\mathrm{T}, \mathrm{EF}}$ than total emissivity $\mathrm{E}_{\mathrm{T}}$ of real area of cavity $\mathrm{A}_{\mathrm{r}}$. Effective emissivity rising up with the increase of ratio $\gamma_{\omega}=A_{r} / A_{o}$.

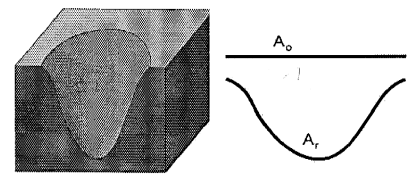

Figure 1: Cavity - cross-section of a surface.

Emissivity of any body, due to the physical and chemical processes resulting from temperature and humidity changes as well as time, constantly changes. A characteristic feature, which is independent upon type of material, is changing 
emissivity and roughness. Generally speaking emissivity increases with the increase of surface roughness. If it is assumed that the roughness consist of set of micro cavities and the measure of unevenness is represented by parameter $\gamma_{\omega}$, then $\mathrm{E}_{\mathrm{T}, \mathrm{n}}$ is changing according to the equations given below:

$$
E_{T, n}=\frac{E_{T, n, 0} \sqrt{1+4\left(\frac{R_{y}}{S_{m}}\right)^{2}}}{1+\left(\sqrt{1+4\left(\frac{R_{y}}{S_{m}}\right)^{2}}-1\right) E_{T, n, 0}}
$$

or

$$
E_{T . n}=\frac{E_{T, n, 0}}{1+\left(\gamma_{\omega}-1\right) E_{T, n, 0}}
$$

where: $\mathrm{R}_{\mathrm{y}}-$ average height of unevenness,

$\mathrm{S}_{\mathrm{m}}-$ average spacing of unevenness,

$\mathrm{E}_{\mathrm{T}, \mathrm{n}, \mathrm{o}}$ - total emissivity in normal direction.

The above equation are true regardless the type of material. Parameter $\gamma_{\omega}$ can be a measure of surface roughness since it is a function of parameters characterising geometrical microstructure of surface $\mathrm{R}_{\mathrm{y}}$ and $\mathrm{S}_{\mathrm{m}}$.

For the purpose of microstructural analysis of surfaces SEM BS300 has been used. The magnified profile of micro-roughness of surfaces has been obtained by "modulation Y". In principle it is based on application of proportionality between vertical deviation of lines and brightness of scanned images. Brightness depends on chemical composition of analysed layer and geometrical microstructure. Therefore, for the constant chemical composition of surface layer, any deviation of line depends only on geometrical microstructure. Value of magnification in direction $\mathrm{Y}$ requires therefore calibration. Cavity can be also determined in simplified way by using the ratio below:

$$
\gamma_{\omega}=\mathrm{L}_{\mathrm{r}} / \mathrm{L}_{\mathrm{o}}
$$

where: $L_{r}-$ real length of the profile,

$\mathrm{L}_{\mathrm{o}}$ - geometrical length of the profile.

The method, therefore, is based on the measurement of real length of profile on the specified section of geometrical length.

\section{Material tested}

The experiment has been performed on surfaces of glass samples (BK7). Samples have been prepared in form of discs with diameter $50 \mathrm{~mm}$ and thickness of $4 \mathrm{~mm}$. Surfaces have been sanded with sand of different size particles resulting in different roughness - 100 (sample no 1), 150 (sample no 2), 360 (sample no 3), 500 (sample no 4) and 700 (sample no 5). Roughness of the samples have 
been assessed by contact method, optical method and after covering with thin layer of gold also by modulation Y method, with application of SEM.

Physical properties of glass BK7:

- density: $2510 \mathrm{~kg} / \mathrm{m}^{3}$

- average coefficient of linear expansion in temperature rage 293-393K:

$7.1 \cdot 10^{-6} \mathrm{~K}^{-1}$

- specific heat under the constant pressure $(0.10135 \mathrm{MPa})$ and temperature 299K: $858 \mathrm{~J} / \mathrm{kgK}$

- coefficient of thermal conductivity in temperature $293 \mathrm{~K}: 1.10 \mathrm{~W} / \mathrm{mK}$

- optical transmittance in range 0.37-1.5 $\mu \mathrm{m}: 0.1 \mathrm{~cm}^{-1}$

- coefficient of light refraction for wave $0.6328 \mu \mathrm{m}: 1.51509$

- temperature coefficient of light refraction for wave $0.6439 \mu \mathrm{m}$ in the temperature range $213-293 \mathrm{~K}: 0.5 \cdot 10^{-6} \mathrm{~K}^{-1}$

\section{Experimental results}

Analysis of geometrical microstructure of samples surfaces showed clear differences between roughness parameters determined by three methods. Different methods characterised with different level of accuracy.

Results roughness parameters obtained by contact method for five different glass surfaces varied significantly. The length of elementary section $\mathrm{L}_{c}$ was $0.70 \mathrm{~mm}$.

The main parameters of measured profile are shown in Table 1.

In optical method experiment was based on determination of such an angle $\Theta$ for which the mirror component is not seen on the background of diffusive component. This enabled determination of the mean square deviation of profile and mean arithmetic deviation of profile. The results of surface roughness obtained form optical method are presented in Table 2.

Application of the Scanning Electron Microscope allowed to analyse not only the images of different surfaces, but also randomly selected three profiles in a central part of sample, obtained by modulation Y method. The values of cavities $\gamma_{\omega}$ have been determined for different profiles $\mathrm{Y}$ and presented in Table 3.

Table 1: $\quad$ Main parameters measured by contact method.

\begin{tabular}{|c|c|c|c|c|c|c|c|c|}
\hline $\begin{array}{c}\text { Surface } \\
\text { No. }\end{array}$ & $\begin{array}{c}\mathrm{R}_{\mathrm{a}} \\
{[\mu \mathrm{m}]}\end{array}$ & $\begin{array}{c}\mathrm{R}_{\mathrm{q}} \\
{[\mu \mathrm{m}]}\end{array}$ & $\begin{array}{c}\mathrm{R}_{\mathrm{y}} \\
{[\mu \mathrm{m}]}\end{array}$ & $\begin{array}{c}\mathrm{R}_{\mathrm{tm}} \\
{[\mu \mathrm{m}]}\end{array}$ & $\begin{array}{c}\mathrm{R}_{\mathrm{pm}} \\
{[\mu \mathrm{m}]}\end{array}$ & $\begin{array}{c}\mathrm{S}_{\mathrm{m}} \\
{[\mu \mathrm{m}]}\end{array}$ & $\begin{array}{c}\Theta_{\mathrm{w}} \\
{[\mathrm{deg}]}\end{array}$ & $\begin{array}{c}\mathrm{m}_{\mathrm{w}} \\
{[1 / \mathrm{mm}]}\end{array}$ \\
\hline 1 & 3.90 & 4.80 & 26.00 & 22.00 & 10.00 & 10.7 & 36 & 36 \\
\hline 2 & 3.12 & 3.88 & 20.40 & 17.60 & 9.40 & 3.90 & 58 & 45 \\
\hline 3 & 1.32 & 1.71 & 10.10 & 8.80 & 3.90 & 1.23 & 65 & 57 \\
\hline 4 & 0.91 & 1.15 & 6.88 & 6.26 & 2.42 & 0.55 & 73 & 93 \\
\hline 5 & 0.55 & 0.68 & 4.08 & 3.58 & 1.58 & 0.10 & 85 & 84 \\
\hline
\end{tabular}


Table 2: $\quad$ Main parameters measured by optical method.

\begin{tabular}{|c|c|c|c|}
\hline Surface No & $\begin{array}{c}\Theta_{\mathrm{o}} \\
{[\mathrm{deg}]}\end{array}$ & $\begin{array}{c}\sigma \\
{[\mu \mathrm{m}]}\end{array}$ & $\begin{array}{c}\mathrm{R}_{\mathrm{a}} \\
{[\mu \mathrm{m}]}\end{array}$ \\
\hline 1 & 88.50 & 5.81 & 4.64 \\
\hline 2 & 88.00 & 4.52 & 3.61 \\
\hline 3 & 86.50 & 2.11 & 1.68 \\
\hline 4 & 84.00 & 1.82 & 1.45 \\
\hline 5 & 78.00 & 1.12 & 0.89 \\
\hline
\end{tabular}

Table 3: $\quad$ Cavities measured by SEM method.

\begin{tabular}{|c|c|c|c|}
\hline Surface No & $\gamma_{\omega 1}$ & $\gamma_{\omega 2}$ & $\gamma_{\omega 3}$ \\
\hline 1 & 4.20 & 3.42 & 3.46 \\
\hline 2 & 4.33 & 3.81 & 4.12 \\
\hline 3 & 4.56 & 4.44 & 5.27 \\
\hline 4 & 4.02 & 4.64 & 4.08 \\
\hline 5 & 4.38 & 5.08 & 4.90 \\
\hline
\end{tabular}

\section{Discussion and final remarks}

Based on the experimental results it is possible to identify set of parameters of particular importance. It has been assumed that the following parameters can be used to describe geometrical microstructure of surfaces:

mean arithmetic deviation of profile $\mathrm{R}_{\mathrm{a}}$; mean square deviation of profile from the mean line $R_{q}$; height of the profile peaks measured by ten points $R_{y}$; maximum height of profile peaks $\mathrm{R}_{\max }$; mean spacing between profile peaks $\mathrm{S}_{\mathrm{m}}$; average angle of peaks inclination $\Theta_{\mathrm{w}}$; number of peaks in the profile $\mathrm{m}_{\mathrm{w}}$.

From the above group it is possible to select certain parameters particularly useful from practical point of view. Comparison of the results obtained from contact method: $R_{a}, R_{y}, R_{q}, R_{t m}$ and $R_{p m}$ (see Fig. 2) give clear evidence of variation in values for different samples. Vertical parameters of profile are usually characterised by mean arithmetic deviation of profile $R_{a}$ and mean square deviation of profile from mean line $\mathrm{R}_{\mathrm{q}}$.

In the case of the roughness assessment by optical method, firstly the average value of unevenness $h$ is experimentally determined, and then it is possible to estimate $R_{a}$ and $\sigma$, which is effectively equal to $R_{q}$. The results have been presented in Figure 3. 


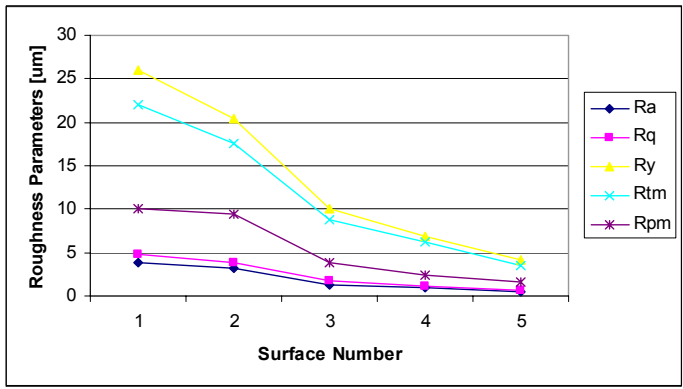

Figure 2: Values of the main roughness parameters - contact method.

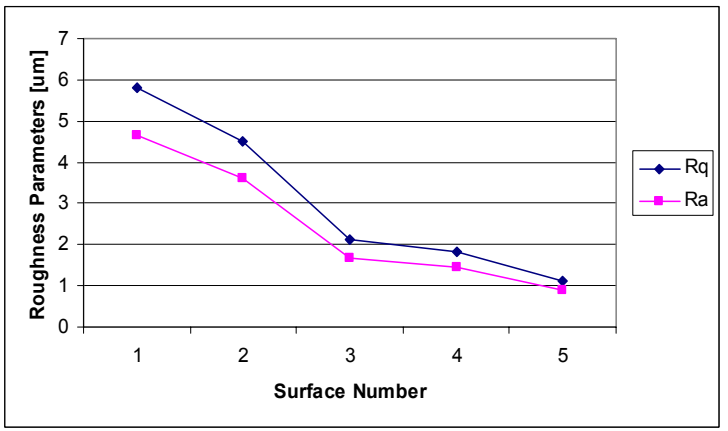

Figure 3: Values of the main roughness parameters - optical method.

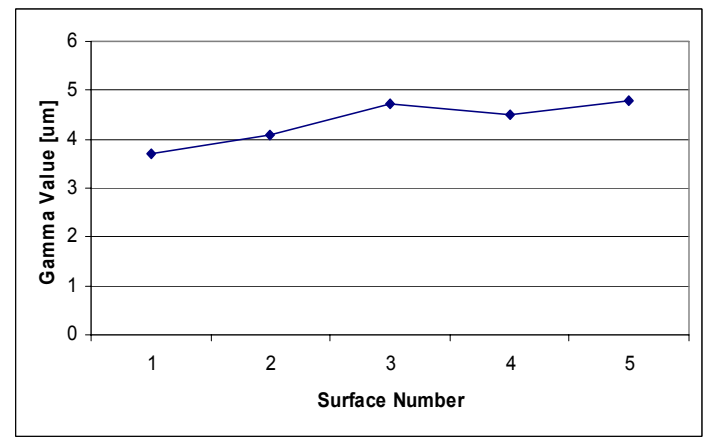

Figure 4: Roughness parameter "gamma" - modulation Y method.

Results obtained from microscopic method very clearly confirm the variations in roughness characteristics of tested surfaces and can be successfully used in comparative studies (Fig. 4). 
The measurements in the Modulation $\mathrm{Y}$ method are restricted to determination of parameter $\gamma_{\omega}$, which is the ratio of the real profile length to the geometrical length of profile, and is not a sufficient on its own to describe the geometrical microstructure. The following average results for samples 1 to 5 have been recorded: $3.69 ; 4.09 ; 4.72 ; 4.49$ and 4.79 . With the exemption of surface no. 4 the cavity values showing a tendency to progressive increase, giving the same an evidence of more and more developed surfaces.

The main disadvantage of the method for considered application is the fact that the method does not allow direct measurement of parameters such as $R_{a}, R_{q}$, $\mathrm{S}_{\mathrm{m}}, \Theta_{\mathrm{w}}$ and therefore it is not recommended on its own for analysis of the condensation process kinetics.

Parameter $\mathrm{R}_{\mathrm{a}}$ determined by both contact and optical methods seems to be of the similar order. Figure 5 presents the relationship between values of $R_{a}$ obtained for these methods.

Figure 6 presents the average results of mean distance between unevenness $\mathrm{S}_{\mathrm{m}}$, average angle of peaks inclination $\Theta_{\mathrm{w}}$, and number of peaks in the profile $\mathrm{m}_{\mathrm{w}}$ obtained from contact method.

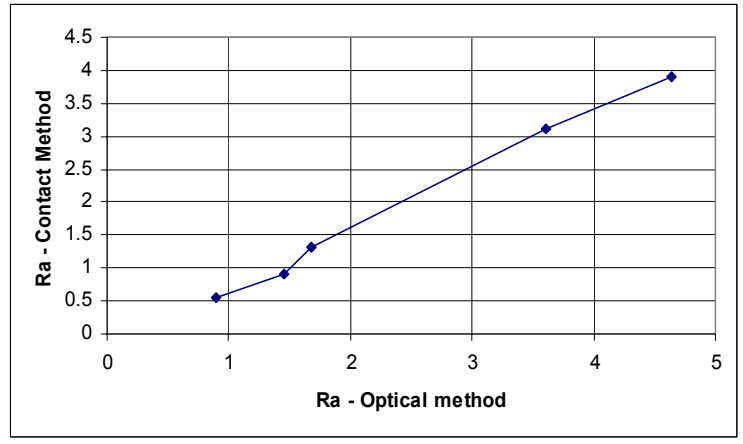

Figure 5: Comparison of parameter $\mathrm{Ra}$ measured by contact and optical methods.

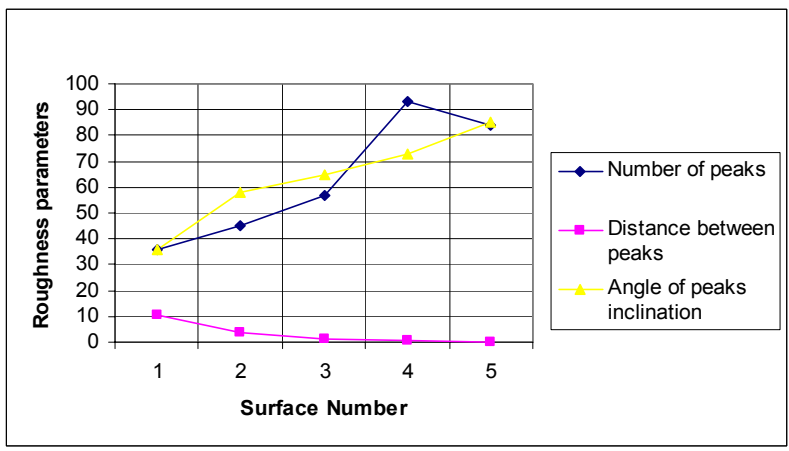

Figure 6: Roughness parameters from contact method. 
Number of peaks in a profile $\mathrm{m}_{\mathrm{w}}$ plays an important role in the overall assessment of surfaces from the point of view of likelihood of water vapour condensation. However in the analysis of the kinetics of individual nucleus creation, $\mathrm{m}_{\mathrm{w}}$ is only of the secondary importance.

\section{References}

[1] CIBSE guide. "Moisture transfer and condensation" CIBSE 1986

[2] Klemm P., Gawin D., "Approximated Mathematical Model of Coupled Transport of Mass and Heat in Building Partitions” CPBP 02.21.1.27, Lodz, (1987) (in Polish)

[3] Klemm AJ, Klemm P, Rozniakowski K, Galbraith G H "Non-contact methods of measuring moisture concentrations in external layers of building partitions. I - The influence of geometrical microstructure on the kinetics of moisture condensation on glass surfaces" Building and Environment, Vol.37, No12, pp. 1215-1220, 2002

[4] Klemm AJ, Klemm P, Rozniakowski K, Wojtatowicz T "Non-contact methods of measuring moisture concentrations in external layers of building partitions. II - Monitoring of the water vapour condensation on porous surfaces" Building and Environment, Vol.37, No12, pp.1221-1232, 2002

[5] Drobnik A., Rozniakowski K., Wojtatowicz T.W., "Contactless investigations of the porous materials with the aid of a He-Ne laser light" Kv.Elektr., Vol.22, no.7, (1995), p.741

[6] Rozniakowski K, Klemm P, Klemm A J "Some experimental results of laser beam interaction with surface layer of brick" - Building and Environment, Vol. 36, No.4, pp. 485 - 491, 2001

[7] Rozniakowski K., "Laser investigations of electrotechnical steel surface roughness", Optica Applicata, vol.22 (1992), pp.137-142

[8] Beckmann P., Spizzichino A., "The scattering of electromagnetic waves from rough surfaces", Pergamon Press, New York, 1963 